Revue d'histoire de l'Amérique française

RAS REVUE D.HISTOIRE DE L'AMÉRIQUE FRANÇAISE

\title{
Les rééditions de l'Histoire du Canada de François-Xavier Garneau devant la critique, 1913-1946
}

\section{Pierre Savard}

Volume 28, numéro 4, mars 1975

URI : https://id.erudit.org/iderudit/303394ar

DOI : https://doi.org/10.7202/303394ar

Aller au sommaire du numéro

Éditeur(s)

Institut d'histoire de l'Amérique française

ISSN

0035-2357 (imprimé)

1492-1383 (numérique)

Découvrir la revue

Citer cet article

Savard, P. (1975). Les rééditions de l'Histoire du Canada de François-Xavier Garneau devant la critique, 1913-1946. Revue d'histoire de l'Amérique française, 28(4), 539-553. https://doi.org/10.7202/303394ar d'utilisation que vous pouvez consulter en ligne. 


\title{
LES RÉÉDITIONS DE L'HISTOIRE DU CANADA DE FRANÇOIS-XAVIER GARNEAU DEVANT LA CRITIQUE, 1913-1946
}

\author{
Pierre Savard \\ Centre de recherche en \\ civilisation canadienne-française \\ Université d'Ottawa
}

Dès sa parution en 1845 , le premier tome de l'Histoire $d u$ Canada de François-Xavier Garneau a suscité des polémiques. Tout en reconnaissant ses grands mérites, des critiques, surtout ecclésiastiques, n'ont pas manqué de relever au cours de l'ouvrage des passages empreints à leurs yeux de gallicanisme, de libéralisme voire de "protestantisme". Dans les éditions ultérieures, l'auteur a atténué certaines affirmations. Et surtout, ses thuriféraires comme l'abbé Casgrain se sont efforcés de présenter de lui une image rassurante. En sorte qu'avec le temps, le côté anticléricale de l'Histoire de Garneau a été quelque peu oublié au profit il est vrai de son esprit nationaliste canadien-français. Comme si, dans la société ultramontaine qu'est le Canada français de la fin du $19 \mathrm{e}$ siècle, le patriote faisait pardonner les écarts du libéral de 1830. Toutefois, au début de ce siècle, la réédition de l'Histoire $d u$ Canada par son petit-fils Hector va remettre en cause ce délicat équilibre et rouvrir un débat qui durera trois longues décennies.

C'est autour de 1900 que Hector Garneau, fils d'Alfred, entreprend de préparer une nouvelle édition de l'Histoire $d u$ Canada. Il va réaliser le rêve de François-Xavier Garneau de publier à Paris. En 1913, paraît le premier des deux tomes de la cinquième édition. A cause de la Grande Guerre, le second tome ne sera publié qu'en 1920.

Hector Garneau est né à Ottawa le 9 juin 1872 du mariage de Alfred, fils de l'historien Francois-Xavier Garneau (18091866) et de Elodie Globensky dont la famille est liée aux Papineau. Son père Alfred (1836-1904) est traducteur au Sénat et poète à ses heures. Hector Garneau étudie au Collège de l'Université d'Ottawa, puis à l'Université Laval de Montréal où il fait son droit. Il est admis au Barreau en 1895. Délaissant la pratique 
du droit, il se livre au journalisme et à la littérature. Déjà en 1892 il avait tenu une rubrique au Monde de Montréal intitulée "Heures de causeries". Pendant deux ans il remplit la fonction de chef adjoint des nouvelles à la Patrie. En 1903 lors de la fondation du Canada il assume la codirection de l'organe libéral jusqu'en 1904. En 1904-1905 on le retrouve chef de cabinet de Louis-Philippe Brodeur, ministre du revenu dans le gouvernement Laurier. En 1906 il réunit les volumes de son père sous le titre Poésies d'Alfred Garneau. Alfred DeCelles le recommande au poste de conservateur de la Bibliothèque municipale de Montréal en ces termes en 1909: "Durant plusieurs années, je l'ai vu à l'œuvre à la bibliothèque du Parlement et j'ai pu constater l'étendue de ses connaissances en bibliographie. Je n'ai jamais rencontré un homme de son âge mieux renseigné en histoire, en économie politique, en science sociale. Homme du monde accompli, parlant l'anglais avec la même aisance que le français..." 1

Hector Garneau déploie une grande énergie dans la mise à jour de l'Histoire. Il multiplie les précisions et les rectifications dans quelque deux mille notes et deux cents appendices tout en respectant scrupuleusement le texte de son grand-père. Mais pour une raison que nous ignorons, Hector Garneau décide de revenir au texte de la première édition de $1845-1852$, ce qui ne va pas manquer de susciter bien des difficultés. Le texte de cette édition, on l'a vu, est considéré plus osé au point de vue des jugements en matière politico-religieuse.

Le premier tome qui sort des presses en 1913 arrive dans une conjoncture favorable. Le renouveau nationaliste des années

${ }^{1}$ Lettre de DeCelles au Secrétariat de la Commission municipale de Montréal, Ottawa, 4 mars 1909. Cette lettre de même que tous les autres manuscrits cités et la plupart des coupures de presse et des articles de revues sont conservés dans le fonds Garneau (1809-1954) déposé au Centre de Recherche en civilisation canadienne-française de l'Université d'Ottawa par M. Edouard Garneau, arrière-petit-fils de l'historien national et fils d'Hector Garneau. Le 18 janvier 1905, Hector Garneau a épousé à Montréal Blanche Pillet (1877-1933), fille de l'avocat Henri Pillet et de Georgiana De Lisle dont il aura deux fils, Edouard et De Lisle Garneau. En 1916, il devient conservateur de la bibliothèque de la Ville de Montréal. Il occupera le poste jusqu'à sa retraite en 1930. Hector Garneau se révèle un fidèle partisan et un ami de Laurier, comme en témoigne leur correspondance en partie conservée dans les archives familiales. Il est aussi lié à Henri Bourassa (sa mère est une Globensky apparentée aux Papineau). Lors de la scission Laurier-Bourassa, Hector Garneau reste fidèle au premier. Il meurt à Montréal le 21 mai 1954 en sa 83e année. Sur Hector Garneau, voir la notice de la Revue du Barreau (vol. 14, no 6 (juin 1954) : 327-328) par Jean-Jacques et Paul Lefebvre. L'annonce de son décès a paru dans Le Devoir, La Presse, le Star et la Gazette de Montréal du 23 mai 1954 avec une brève biographie. Voir aussi les diverses éditions du Who's who in Canada entre 1930 et 1954. 
1900 a ravivé le souvenir jamais éteint de Garneau. Dans le domaine de l'éducation, Charles-Joseph Magnan s'est fait le propagateur de l'Histoire du Canada insistant sur ses aspects nationaliste et conservateur. En 1909, on a rappelé le centenaire de la naissance de l'historien ${ }^{2}$. En 1912 des fêtes grandioses à Québec ont eu lieu à l'occasion du dévoilement du monument offert par l'industriel Georges-Elie Amyot. On y a vu sir François Langelier, lieutenant-gouverneur du Québec, aux côtés du premier ministre sir Lomer Gouin et de sir Wilfrid Laurier. Hector Garneau, invité à parler, a rappelé "les enseignements de Garneau" "à une époque où le fanatisme ancien veut de nouveau menacer tout ce qui porte une marque française en ce pays..." L'abbé Amédée Gosselin, supérieur du Séminaire de Québec, y a fait l'éloge de Garneau tout en exprimant des réserves au sujet des premières éditions ${ }^{3}$.

La critique parisienne, dès la parution, multiplie les comptes rendus favorables au premier tome de la nouvelle édition de l'Histoire du Canada. Un service de presse bien organisé, l'appui du comité France-Amérique et une préface de Gabriel Hanotaux, grande personnalité du monde politique français du temps, aident à comprendre l'éclat de cette parution. Les deux mille exemplaires de l'édition originale sont épuisés en six mois. Les critiques comme Paul Kaeppelin de la Revue d'histoire moderne et contemporaine s'accordent généralement pour louer la qualité scientifique du travail du petit-fils. Gabriel Hanotaux, pour sa part, parle de "véritable encyclopédie de l'histoire du Canada". "Acte de haute et bienfaisante politique", souligne André Siegfried dans la Revue politique et parlementaire, en songeant au renforcement des liens entre les deux pays ${ }^{4}$. Dans sa copieuse préface de vingt-trois pages, Hanotaux s'étend sur "la leçon du Canada". Il blâme le gouvernement des $17 \mathrm{e}$ et $18 \mathrm{e}$ siècles qui "marchanda toujours l'existence" à la Nouvelle-France et "n'eut jamais confiance en son avenir". Devant les résultats piteux de cette politique coloniale, "le regret le dispute au remords", de s'écrier Hanotaux. Et l'ancien ministre des Affaires étrangères

2 Voir par exemple la une de La Tribune de Saint-Hyacinthe du 18 juin 1909 sous la signature de Julien Brieux qui suggère l'érection d'un monument. de Québec.

3 Le Devoir du 21 octobre 1912, en première page. Voir aussi Le Soleil

4 Les archives de la famille Garneau conservent un dossier considérable de ces comptes rendus souvent recueillis par l'Argus de la Presse de Paris et sans doute transmis à Hector Garneau par la maison Alcan. Voir un choix de commentaires louangeurs dans Le Canada du 6 juin 1914, p. 4 (page éditoriale). 
de se demander si la France, instruite de "la leçon du Canada", saura en 1913 garder et développer ses nouvelles colonies? Cette utilisation de l'histoire canadienne par le "lobby impérialiste" français aide aussi à comprendre le bruit que le livre fait en France, dans des périodiques tels que les Questions diplomatiques et coloniales, la Quinzaine coloniale ou la Revue d'histoire des colonies françaises.

En Amérique, le livre est plus diversement accueilli. Le professeur Colby de McGill en fait l'éloge dans l'American Historical Review tandis que le professeur W. L. Grant en dit du bien dans la Review of Historical Publications relating to Canada. Même témoignage favorable dans The Nation de New York. Le Canadien Léon Gérin se félicite de la publication dans La Science sociale de Paris ${ }^{5}$.

Mais nombreuses sont les critiques venant du Canada français. La Vérité de Québec, dirigée par Paul Tardivel, fils de Jules-Paul Tardivel (décédé en 1905), met vite ses lecteurs en garde contre la nouvelle édition. Le 8 novembre 1913 elle offre à ses lecteurs la reproduction de la première partie d'une étude du jésuite Armand Chaussegros parue dans le Messager canadien $d u$ Sacré-Cœur de Jésus. Le critique ecclésiastique s'en prend en particulier à un passage de l'introduction où est affirmée sans ambages la suprématie du pouvoir civil sur le pouvoir religieux. Le passage incriminé, dû à la plume d'Hector Garneau, se lit comme suit: "Mais il [François-Xavier Garneau] met en lumière les intérêts supérieurs de l'Etat et la prédominance qui revient au pouvoir civil "." Et le clerc de répéter l'accusation de gallicanisme, cette fois à l'endroit du petit-fils de l'historien national. 'D'où vient donc, se demande le critique, que les protestants en général ne tarissent pas d'éloges sur cette histoire et que la presse radicale la recommande chaudement? ${ }^{7}$ C'est que Garneau "descendant de huguenots a souvent fait écho aux historiens de ce parti et que formé à l'école de Voltaire et de Michelet il avait d'étranges et confuses idées sur la liberté et les principes de la révolution de 1789" 8 . Puis le jésuite de reprendre

5 Extraits de ces témoignages dans Le Canada (article cité) qui fait un accueil sans réserve à l'ouvrage. La plupart des articles sont conservés in extenso dans les archives de la famille Garneau.

6 Ce passage sera remanié comme suit dans l'édition de 1928: "Surtout il met en relief les intérêts dominants de l'Etat et l'indépendance absolue du pouvoir civil."

7 Allusion sans doute au Canada qui représente la tendance la plus à gauche du parti libéral.

8 L'argument des ancêtres huguenots en France au début du $17 \mathrm{e}$ siècle apparaît mince. Comme d'autres colons venus en Nouvelle-France, Louis Garneau a pu être un protestant passé au catholicisme. 
les principaux jugements et récits de Garneau qui prêtent à controverse. Le 29 août 1913, "un professeur d'histoire" avait déjà publié, dans l'Action sociale de Québec, un long article intitulé "Quelques remarques sur l'Histoire du Canada de François-Xavier Garneau”. L'auteur dénonce lui aussi le "drapeau gallican" que déploie hardiment Hector Garneau. Il reprend le procès de François-Xavier Garneau disciple de Voltaire et de Michelet. Il s'attache à refuser l'affirmation de Garneau à savoir que le peuple est la source de tout pouvoir. Il ne manque pas de blâmer la tolérance de Garneau en faveur des huguenots et plus encore ses lignes énergiques blâmant Mgr de Laval. Il termine enfin sèchement: "Malgré son fatras de notes et d'appendices, au point de vue de la critique historique, cet ouvrage est une régression."

Le 26 novembre, c'est au Devoir d'entrer en scène. Henri Bourassa se réjouit de l'idée qu'on a eue de rééditer Garneau et félicite Hector Garneau de son bon travail. Mais l'essentiel de son article constitue une critique de la préface de Hanotaux trop sévère à son gré envers les dirigeants de la France de l'Ancien Régime. Bourassa défend ici une idée qui lui est chère à savoir que les grandes œuvres de la France ne commencent pas en $1789^{\circ}$. L'article a le don de réjouir la presse d'extrêmedroite française. C'est ainsi que L'Action française du 21 décembre 1913 en reprend l'essentiel sous la plume de Antoine Lestra ${ }^{10}$.

Le 1er décembre 1913, Hector Garneau adresse en réponse une longue lettre publiée dans Le Devoir du 5 de ce mois. Il explique que l'admiration de son grand-père s'adressait plus à Voltaire historien qu'à Voltaire philosophe. Puis il défend le prétendu gallicanisme de Garneau au nom de la tolérance en terminant avec le mot de Brunetière: "Si nous voulons que l'on respecte la liberté de notre conscience, il nous faut commencer par respecter celle des autres." Dans le même numéro Henri Bourassa discute pied à pied les affirmations de son correspondant. Il refuse la distinction de Voltaire, déclarant de façon péremptoire: "Il faut être pour ou contre Voltaire". Puis il sert à Hector Garneau une leçon sur le gallicanisme et ses méfaits

9 Dans la livraison du lendemain, Bourassa s'en prend aux mauvais maîtres de Garneau, Michelet et Voltaire, dénonce les tendances gallicanes de Garneau et déplore que l'historien ait regretté que Richelieu ferme la colonie aux huguenots.

10 Dans L'Univers, Gustave Gautherot parle de l'édition de 1913. Après avoir fait des réserves d'usage sur les idées de Garneau il s'étend sur "la leçon du Canada", à la façon de Hanotaux (reproduit dans L'Action sociale de Québec du 24 septembre 1913). 
dans l'histoire. Enfin il termine en disant que, tout en ne partageant pas bien des opinions de François-Xavier Garneau, il n'en admire pas moins "la bonne volonté, le patriotisme et la studieuse persévérance" de l'historien national.

La presse radicale ne manque pas de souffler sur le feu en défendant le choix d'Hector Garneau de rétablir le texte primitif de l'Histoire $d u$ Canada de préférence au texte de 1859, dernière édition du vivant de l'auteur. Dans un article publié en décembre 1913, dans le Pays de Montréal, "Junius" se félicite qu'on ait mis de côté le "texte expurgé" pour revenir à la première édition ${ }^{11}$. En guise de réponse, le Bulletin du Parler français reproduit quelques lignes de Henri Froidevaux, professeur à l'Institut catholique de Paris, parues dans Polybiblion de juillet 1913 sur le tome premier de l'Histoire du Canada: "Cette Histoire $d u$ Canada est-elle bien celle qu'eût écrite François-Xavier Garneau s'il eût vécu à notre époque? Aurait-il approuvé la manière d'agir de son petit-fils qui a été de reprendre dans les premières éditions des pages supprimées par l'auteur? C'est une question qu'il est permis de se poser?"' 12

A la fin de mai 1914, il est question d'élire Hector Garneau à la Société royale du Canada. Selon un journal libéral de Montréal, le Bulletin du 7 juin 1914, "la faction de Québec" de la société dans l'esprit des gens de l'Action Sociale et de la Vérité fait en sorte que le petit-fils de l'historien ne soit pas admis ${ }^{13}$. Au printemps suivant, les portes de l'Académie canadienne sont ouvertes à Hector Garneau. C'est Rodolphe Lemieux qui le présente et fait son éloge ${ }^{14}$. L'élection n'a pas été sans difficulté. François Langelier, dans une lettre à Hector Garneau du 9 mars, lui apprend qu'il faut compter avec l'opposition de Mgr Camille Roy et de Adjutor Rivard. Selon Langelier, Mgr Bruchési a fait campagne contre la candidature d'Hector Garneau ${ }^{15}$.

${ }^{11}$ L'hebdomadaire, Le Pays, est l'organe de l'aile gauche du parti libéral de 1910 à 1921. Il tire à 11,500 exemplaires en 1913. Mgr Bruchési vient de le condamner le 25 septembre 1913 pour ses positions favorables à la libre-pensée.

${ }_{12}$ Le Parler français. Bulletin de la Société du Parler français, XIII, no 3 (novembre 1914) : 127, signé A. R. [Adjutor Rivard].

$13 \mathrm{Il}$ aurait fallu pour ce faire suspendre les règles parce qu'il y avait deux candidats pour une nomination. Mgr Bégin et Mgr Bruchési avaient été admis suivant cette procédure, de souligner l'organe radical.

${ }_{14}$ La Presse, 27 mai 1915, 7. Voir aussi Le Canada du 6 avril 1915, 4 (signé F. R.). La Presse du 5 avril annonce son élection. Le Pays du 10 avril, sous la plume de Paul S. Bédard, fait l'éloge du "libéralisme qu'il [Hector Garneau] incarne". Ce qui n'est pas une recommandation dans les milieux cléricaux et conservateurs.

15 F. Langelier à Hector Garneau, Spencer Wood, 9 mars 1914. 
En 1920 paraît à Paris le second tome de l'Histoire $d u$ Canada préparé par Hector Garneau avant 1913 et dont la guerre a empêché la sortie. En même temps, la maison Alcan lance une 6e édition. En 1928, sort des mêmes presses une 7e édition. Hector Garneau y a fait des changements mineurs dans son introduction, mais l'œuvre reste identique ${ }^{16}$.

Durant les années 1920 et 1930 l'œuvre de François-Xavier Garneau continue d'intéresser la critique. Henri d'Arles [l'abbé Henri Beaudé] la présente avec chaleur dans Nos historiens: Cours de critique littéraire professé à Montréal sous les auspices de l'Action française (Montréal, 1921). En 1926, Gustave Lanctot publie son François-Xavier Garneau à Toronto, dans la collection "Makers of Canada series". L'étude légèrement remaniée sera reprise chez Fides à Montréal en 1946. Si ces auteurs se mettent aisément d'accord pour célébrer le patriotisme de Garneau, les critiques de ses jugements religieux ne désarment pas. Entre 1924 et 1927, l'abbé Georges Robitaille, professeur au Collège classique de Joliette, publie des articles dans Le Canada français en vue de rectifier des jugements de Garneau ${ }^{17}$. En avril 1929 Le Canada français publie un article de l'abbé Napoléon Morissette intitulé "En marge des nouvelles éditions de Garneau". Le prêtre-professeur d'histoire au Séminaire de Québec reproche à Hector Garneau d'avoir publié l'édition de 1928 (la septième) sans tenir compte de la critique.

Pour dissiper tout malentendu, Hector Garneau prie la revue d'insérer une note dans laquelle il explique que les éditions de 1920 et 1928 ne furent à toutes fins utiles que des réimpressions. “Tout au plus les éditeurs m'ont-ils permis de changer quelques lignes dans mon Introduction", précise-t-il. Puis il annonce la préparation d'une édition nouvelle de l'Histoire du Canada. "Cette édition sera claquée sur la quatrième et, nous en avons l'espoir, ne prêtera plus aux controverses." Délestée d'une grande partie de l'appareil critique des éditions précédentes, elle sera destinée "au grand public, aux élèves de nos couvents et de nos collèges". "Ainsi présenté", espère le petit-fils de FrançoisXavier Garneau, l'ouvrage "fera davantage appel à nos compatriotes à qui elle continuera d'enseigner, avec le culte du passé,

16 Plus précisément 15 passages ont été modifiés pour des raisons en général stylistiques. Le seul changement significatif est celui concernant les relations des pouvoirs civil et religieux mentionnés plus haut. Dans une lettre à Mgr Camille Roy du 18 décembre 1928, Hector Garneau précise que la septième édition (de 1928) n'est que la réimpression des deux précédentes à l'exception de la correction du passage de la p. XXXVI.

17 Repris dans Etudes sur Garneau (1929). 
la fidélité à notre langue, notre religion, nos lois, une foi invincible en nos destinées ${ }^{18}$."

Le 20 avril 1929 Hector Garneau adresse à l'abbé Napoléon Morissette une lettre dans laquelle il explique que ni lui-même ni son aïeul n'ont quelque parti pris contre Mgr de Laval ou le clergé. La lettre constitue un témoignage vibrant des mérites du clergé dans notre histoire. Et l'auteur de préciser: "évêques, prêtres, missionnaires, religieuses, aussi bien que laïques, ensemble nous formons un bloc dont on ne saurait, sans péril extrême pour notre race et sans compromettre notre avenir, détacher aucun élément ni aucune partie 19." La lettre d'Hector Garneau lui vaut une longue réponse du prêtre-historien. L'abbé refait le procès de François-Xavier Garneau ("Le grand historien a pu être sincère; si c'est le cas il faut conclure qu'il a été imparfaitement renseigné."). Mais surtout il malmène le petitfils pour avoir dans ses notes et appendices fait preuve d'une "partialité (qui) crève les yeux" contre Mgr de Laval et le clergé. Et l'abbé de citer les appendices 176, 187, 198, 199 et 202 des $6 e$ et 7e éditions. Plus loin, le professeur d'histoire en Rhétorique au Séminaire de Québec ajoute des remarques intéressantes sur l'utilisation de l'Histoire de Garneau et sur la valeur de l'œuvre. Ces considérations rejoignent sans doute celles de bien des clercs de l'époque: "La quatrième édition de l'Histoire du Canada de votre très distingué aïeul est dans la bibliothèque de classe de mes élèves. Chaque année, je la leur recommande, tout en leur faisant quelques réserves sérieuses sur des points que vous connaissez. Je serais heureux si je pouvais leur mettre entre les mains une édition où ces réserves seraient faites, non pas dans le texte même, mais à l'aide de quelques notes brèves et précises. Cela viendra, vous nous en donnez l'espoir. Abstraction faite de ces réserves, je loue, devant mes jeunes gens, l'œuvre de votre illustre grand-père; j'en fais des éloges en leur faisant voir la belle ordonnance, les tableaux à larges vues et les magnifiques synthèses que nous y trouvons; je leur fais remarquer le style noble et dégagé dans lequel elle est écrite; enfin, j'insiste surtout sur le souffle patriotique qui l'a inspirée du commencement à la fin. Bref, sans être une œuvre parfaite, c'est encore ce que nous avons de mieux comme histoire générale ${ }^{20}$."

Cinq ans plus tard, Hector Garneau qui a pris sa retraite de conservateur de la Bibliothèque municipale de Montréal en

18 Hector Garneau au directeur du Canada français, le 19 avril. Reproduit dans le périodique.

19 Hector Garneau à l'abbé Napoléon Morissette, 20 avril 1929.

20 Napoléon Morissette à Hector Garneau, 26 avril 1929. 
1930, écrit à l'abbé Morissette pour lui annoncer la préparation d' "une réédition entièrement remaniée et mise au point d'après les dernières recherches, et qui sera conforme à la quatrième" (publiée par Alfred Garneau, son père, en 1882). Il se propose d'éliminer à la fois du texte de l'ouvrage et de son introduction qu'il récrira "tout ce qui est sujet à caution ou à critique, et en particulier les opinions premières et surannées de l'auteur". Et il ajoute: "A la vérité, ces opinions étaient de Garneau philosophe et non de Garneau historien, ainsi que l'a fait observer combien justement M. Georges Goyau dans ses Origines religieuses du Canada." Hector Garneau espère voir paraître l'ouvrage à l'automne de 1935 . Il prie son correspondant de faire part de cette lettre à "Son Eminence le Cardinal Villeneuve", archevêque de Québec, en lui rappelant que diplômé du "collège d'Ottawa" il est lui aussi un ancien élève des Oblats de MarieImmaculée ${ }^{21}$.

La préparation de la nouvelle édition prendra plus de temps que prévu à Hector Garneau dont la vue baisse et pour lequel le travail devient de plus en plus pénible. Il attache un soin extrême à présenter une édition qui ne suscite aucune controverse. En 1937, par exemple il adresse à Mgr Camille Roy une lettre de la même encre que celle envoyée à l'abbé Morissette. Il affirme que c'est avec "la plus haute approbation de $\mathrm{Mgr}$ Georges Gauthier, archevêque de Montréal, qu'il prépare sa nouvelle édition entièrement revue et corrigée". "Tous les passages sujets à caution ou à controverse" en seront retranchés. Son introduction et le Discours préliminaire de François-Xavier Garneau vont disparaître. Les notes et appendices feront place à une bibliographie mise à jour et reportée à la fin de chaque volume. De plus, Hector Garneau se propose de développer la partie religieuse "à peine effleurée" par François-Xavier Garneau. Il approuvera le cardinal Richelieu d'avoir fermé la NouvelleFrance aux huguenots. Sur ce point, il peut, dit-il, compter sur l'appui de l'historien Hanotaux. Il va aussi rappeler que l'amiral Coligny fut protestant avant d'être français, suivant en cela Baudrillart plutôt que Raynal comme son aieul. Mais surtout il rendra "pleine justice à la mémoire de Mgr de Laval". Le chapitre intitulé "Luttes de l'Eglise et de l'Etat" aura un titre "moins tendancieux". En résumé, "les ombres philosophiques seront

21 Hector Garneau à Napoléon Morissette, 21 novembre 1934. 
bannies de l'ouvrage et vous n'y trouverez pas, je pense, la moindre teinture libérale, protestante ou gallicane ${ }^{22 " .}$

C'est durant l'été de 1939 que Hector Garneau met la dernière main à sa nouvelle introduction. Mgr Georges Gauthier, archevêque coadjuteur de Montréal auquel il a soumis son texte, le lit attentivement avec un vif intérêt et déclare qu'il est "animé du meilleur esprit ${ }^{23}$ ".

Le projet de l'Histoire se précise de plus en plus dans l'esprit d'Hector Garneau. Dans une lettre à son frère Léon, avocat à Montréal, il explique en 1941 que:

1. Cette réédition diffère de toutes les façons et à tous les points de vue des éditions de Paris;

2. Tous les chapitres ont été remaniés et allongés et les notes et les appendices sont remplacés par une bibliographie mise à jour et renvoyée à la fin de chaque volume;

3. La nouvelle édition, au lieu de reproduire le texte de la première édition de 1845-1848 qui avait soulevé tant d'objections, sera conforme au texte de la quatrième édition de 1882 , publiée par Alfred Garneau. Cette édition de 1882, que j'ai corrigée avec soin, a déjà valu à son auteur, de la part de Mgr Camille Roy, recteur de l'Université Laval, et récemment de Mgr Emile Chartier, vice-recteur de l'Université de Montréal, d'être appelé encore une fois notre historien national; 4. Sur les origines de l'Amérique, sur Jacques Cartier, Champlain, Maisonneuve, Marie de l'Incarnation, Joliette, LaSalle et Frontenac, sur la vie et la carrière de Mgr Laval, sur d'autres personnages de notre Histoire, on trouvera des faits nombreux et des documents originaux, qui n'apparaissent dans aucune des éditions précédentes ${ }^{24}$.

Le Canada français, dans sa livraison de mars 1943, offre en primeur la "Nouvelle introduction à l'histoire du Canada de Francois-Xavier Garneau 25". La Rédaction coiffe le texte d'un avertissement des plus aimables remerciant Hector Garneau du "grand honneur et l'insigne privilège de cette primeur", et ajoute: "Nous voulons dire publiquement à Monsieur Hector Garneau notre profonde satisfaction et notre sincère reconnaissance."

22 Hector Garneau à Camille Roy, 8 mai 1937 (brouillon de lettre). L'accusé de réception de Camille Roy qui communiquera la lettre au cardinal Villeneuve est du 11 mai.

23 Georges Gauthier à Hector Garneau, 21 août 1939. Aussi du même au même, 1er septembre.

24 Hector Garneau à Léon Garneau, Montréal, 8 juin 1941.

25 Le Canada français, XXX, no 7: 481 à 485. 
Hector Garneau apprend au lecteur qu'il a remplacé le Discours préliminaire par une lettre de François-Xavier Garneau à lord Elgin et qu'il a décidé de suivre le texte de 1882 publié par son père Alfred Garneau, "suivant (l'édition) de 1859 que l'historien lui-même avait corrigée et augmentée". "Cependant, continue l'éditeur, une mise au point générale, la suppression de passages périmés et certains redressements s'imposaient. Sans cesser de recourir aux sources, on devait faire état des résultats acquis de l'érudition et en même temps tenir compte des justes observations formulées par la critique. Nous y avons tâché. $\mathrm{Au}$ reste, il nous a semblé nécessaire d'allonger et de continuer le récit, de consacrer une part plus grande aux affaires religieuses, et d'y joindre de rapides esquisses des principaux personnages." Suit un résumé de l'Histoire $d u$ Canada dans lequel Hector Garneau ne ménage pas les louanges à l'endroit de la religion et du clergé. L'avant-dernier paragraphe vaut d'être cité ici: "Et puis enfin nous avons essayé de faire revivre, dans une clarté plus moderne, la figure de l'historien probe, catholique et patriote, dont la pensée dominante se traduisait en maintes pages par ces mots: "notre religion, notre langue, nos lois". Tant il est vrai que pour nous, Canadiens français, sans oublier nos frères acadiens, la foi ancestrale demeure, comme au jour de notre berceau, l'animatrice de nos labeurs, la gardienne de nos traditions, de notre caractère propre, de notre trésor moral, et la garante de l'existence même de notre nationalité." ( $p$. 485)

Le premier tome de la huitième édition de l'Histoire $d u$ Canada sort enfin des presses en mars 1944 aux éditions de L'Arbre à Montréal ${ }^{26}$. Le deuxième tome paraît en avril, le troisième en septembre, le quatrième en novembre, le cinquième en décembre, le sixième en mars 1945 , le septième et huitième en septembre et finalement, le neuvième, qui comprend l'index, en avril $1946^{27}$.

L'accueil de la critique est divisé pour des raisons bien autres que celles des années 1910 et 1920. Dès la parution du premier volume, Léo-Paul Desrosiers publie un bel article dans Le Devoir ${ }^{28}$. Le Canada français de novembre 1945 apporte un

26 Dirigée par Robert Charbonneau et Claude Hurtubise.

27 Nous avons établi ce calendrier approximatif d'après les coupures de presse et la correspondance d'Hector Garneau (critiques et accusés de réception).

${ }_{28}$ Le Devoir, 18 mars 1944. Nous écartons de cette revue les comptes rendus de journaux qui apportent peu au dossier. On trouvera plusieurs coupures de presse dans les archives de la famille Garneau. Voir aussi le numéro de septembre 1944 de Mes Fiches consacré à Garneau. 
article-compte rendu signé Bertrand Lombard (pseudonyme de l'abbé Emile Bégin, professeur de littérature canadienne à l'Université Laval). Le critique passe en revue les premiers cinq tomes. Il se donne pour but de "faire lire le vieil historien, le faire aimer" et il précise: "Les dissertations savantes sur les conditions actuelles de l'histoire, sur les exigences du genre ne sont pas notre fait." (p. 174) Ce qui ne l'empêche pas de souligner que cette édition remaniée, "c'est du Garneau sans en être tout à fait; et quand on voudra lire l'authentique, on devra retourner aux vieilles éditions tant décriées". Le long compte rendu révèle une lecture attentive qui montre les profondes transformations apportées à l'œuvre. Le jeune historien Guy Frégault, déjà connu par ses ouvrages sur La Civilisation en Nouvelle France et Iberville, le Conquérant, présente un compte rendu dans le premier numéro de Lectures. Revue mensuelle de bibliographie critique publiée par la maison Fides ${ }^{29}$. Après avoir souligné que "l'ouvrage de F.-X. Garneau fut le livre du siècle (au Canada)", le critique insiste sur l' "actualité de Garneau", grâce à sa "vigilante lucidité". C'est pourquoi l'œuvre de Garneau n'a pas tellement vieilli. Puis le critique se félicite de la présente réédition faite par Hector Garneau "avec une admirable probité intellectuelle". Tout en louant "sans réserve" le travail, le critique déclare que si le grand public va vers la dernière édition qui lui est destinée, les spécialistes se reporteront sans doute plus volontiers à l'édition de Paris à cause des notes et des appendices, quitte à la corriger et à la compléter avec les données de la huitième édition.

Dans la Revue de l'Université Laval de janvier 1947, Gustave Lanctot présente à son tour un compte rendu à tout prendre très sévère de la huitième édition. Il va même jusqu'à déclarer nettement: "Elle peut sans doute, pour le gros public, former un excellent livre de lecture historique. Pour le lettré et le spécialiste, elle représente une initiative malhabile et malheureuse." (p. 360) Lanctot reproche à Hector Garneau d'avoir supprimé "le remarquable Discours préliminaire, qui figure dans toutes les éditions antérieures". Surtout il regrette que l'éditeur ne s'en soit pas tenu rigoureusement à la dernière édition de François-Xavier Garneau, celle de 1859, qui, en bonne méthode est celle qui "doit rendre témoignage de sa pensée et de sa conscience". Enfin, Lanctot ne manque pas de souligner les contra-

${ }^{29}$ Lectures, 1, no 1 (septembre 1946) : 19-22. 
dictions entre l'édition de Paris de 1913 "de formule radicale" et celle de Montréal de 1944-46 "d'une parfaite orthodoxie ${ }^{30 "}$ ".

Du 23 au 27 avril 1945 a lieu à l'Université de Montréal la Commémoration du Centenaire de la publication de l'Histoire $d u$ Canada. Ottawa et Québec sont aussi la scène de célébrations qui rappellent par leur éclat les grands moments de 1867, lors de la translation des restes de l'historien au cimetière Belmont et l'inauguration du monument de François-Xavier Garneau en 1912 à Québec. Hector Garneau assiste aux fêtes de Montréal. Les critiques de Gustave Lanctot à l'endroit de la huitième édition en cours de parution l'affectent profondément ${ }^{31}$. Prises dans leur ensemble, les communications du Centenaire apportent peu à la connaissance de l'homme et de l'œuvre. Mais ils constituent une excellente occasion de faire connaître Garneau.

La huitième édition semble avoir remporté un beau succès de librairie. Les réserves de Gustave Lanctot et de Jean-Pierre Houle qui tiennent pour le texte de $1859^{32}$ ne passent guère le mur du petit nombre des spécialistes. Hector Garneau reçoit des appuis prestigieux tel celui du vieux sénateur-historien Thomas Chapais ${ }^{33}$. Et surtout, du côté ecclésiastique, la louange est unanime. Le théologien dominicain M.-Ceslas Forest félicite Garneau d'avoir corrigé, dans l'œuvre de son aỉeul, tout ce qui pourrait froisser le sentiment religieux. "Les plus exigeants ne pouvaient attendre davantage de votre part et vous avez montré là un magnifique sentiment chrétien ${ }^{34}$." C'est sans doute du cardinal Villeneuve, archevêque de Québec et successeur de Mgr de Laval que lui vient le témoignage le plus consolant. Le 10 juillet 1944 , le cardinal, sur réception du tome premier, adresse une belle lettre à Hector Garneau dans laquelle il dit:

30 Revue de l'Université Laval, 1 (1946-47): 354-360. La Revue a succédé au Canada français. Lanctot reprend ici les réserves qu'il a exprimées plus brièvement lors de la Semaine d'histoire du Canada. Voir JeanJacques Lefebvre (éd.), Centenaire de l'Histoire du Canada de FrançoisXavier Garneau (...) (Montréal, La Société historique de Montréal, 1945). l'auteur en 1974.

32 Hector Garneau à Guy Frégault, Montréal, 20 septembre 1946. Dans une lettre à Hector Garneau du ler février 1935, Pierre-Georges Roy suggérait de publier une édition "absolument fac simile" de celle de 1859 pour éviter de trahir la pensée de l'auteur.

33 Les papiers d'Hector Garneau renferment quelques billets favorables de Chapais au fur et à mesure de la parution des tomes de la huitième édition. Certains ont été reproduits dans la presse.

34 Forest à Hector Garneau, 10 mai 1946. Voir aussi les félicitations de Mgr Olivier Maurault qui a lu la nouvelle introduction au tome 1 (Maurault à Hector Garneau, Montréal, 27 mars 1944). 
Il serait impertinent de ma part de relever le mérite de l'œuvre de François-Xavier Garneau, et de votre huitième édition. Des connaisseurs l'ont fait unanimement.

Cependant, qu'il me soit permis, à mon titre particulier d'archevêque de Québec, de vous exprimer ma satisfaction que vous ayez compris aussi noblement le dessein patriotique de l'auteur de l'Histoire du Canada, et ayez aussi hautement respecté sa personne.

Car vous avez non seulement rétabli des dates et ajouté des précisions, voire fait des corrections, comme votre aieul, dans son intégrité d'historien, l'eût fait lui-même, par exemple, sur le rôle incomparable de Monseigneur de Laval, mon très vénérable prédécesseur; mais vous avez voulu en outre que, par sa présentation, votre édition puisse contribuer le plus efficacement possible à faire pénétrer chez les nôtres ce sentiment de dignité et cette fierté nationale auxquelles notre histoire nous donne droit, malgré nos malheurs politiques et les fluctuations du temps. Ajouterai-je que vous avez apposé comme un nouveau lustre de clarté et de frâ̂cheur au style de véritable écrivain qui fut celui de François-Xavier Garneau. Car, son œuvre constitue pour nous, au dernier siècle, à peu près l'unique grand œuvre de notre littérature; elle en aura été la pierre d'assise et comme le principe.

A cela vous avez ajouté le soin d'effacer les traces de certaines éclaboussures dont le génie de Voltaire avait trouvé le moyen de souiller dans le temps même les esprits les plus honnêtes. C'est une justice et non pas un redressement que vous aurez ainsi accompli dans l'œuvre admirable de votre grand'père. Ce sont là, Monsieur, les motifs particuliers qui me font apprécier votre labeur personnel pour rendre à l'Histoire du Canada de François-Xavier Garneau tout son éclat et tout son rayonnement ${ }^{35}$.

Cet aperçu de la fortune de l'Histoire du Canada de FrançoisXavier Garneau dans la première moitié du vingtième siècle apparaît riche d'enseignement sur le développement des idéologies et de l'historiographie du Canada français. Le retour à la première édition apparaît nettement une provocation aux yeux de la critique cléricale. Contre l'édition de Paris se sont élevées les mêmes objections qu'avaient suscitées la première édition de Garneau au milieu du $19 \mathrm{e}$ siècle. L'édition de Montréal en 1944-1946 présentera toutes les garanties d'orthodoxie et

35 La lettre a été reproduite dans Le Devoir du 2 septembre 1944. On trouve dans les papiers d'Hector Garneau une autre lettre du 24 avril 1946 où le Cardinal remercie de l'envoi des neuf tomes et exprime des sentiments et des opinions analogues. 
désarmera la critique cléricale. Cependant, l'Histoire de Garneau constitue un monument national que personne ne conteste en bloc malgré des réserves de détail. En 1945 encore, l'ouvrage est même considéré comme une synthèse indispensable sur notre histoire. Cela s'explique non seulement par les qualités intrinsèques de l'œuvre tant au point de vue littéraire qu'à celui de la philosophie de l'histoire nationale, mais encore et surtout par le relatif sous-développement des études historiques d'envergure chez nous. Trente ans plus tard, les progrès des travaux sur la Nouvelle-France et sur le régime anglais ont relégué l'Histoire de Garneau à sa véritable place, non négligeable, celle d'un classique, c'est-à-dire d'un livre d'histoire fait pour être lu plus que consulté. 